\title{
sciendo
}

\section{A Comparison Between the Squat and the Deadlift for Lower Body Strength and Power Training}

\author{
by \\ Federico Nigro ${ }^{1}$, Sandro Bartolomei ${ }^{1}$
}

\begin{abstract}
The aim of this study was to compare the effects of two resistance training programs including either a deadlift or a parallel squat on lower body maximal strength and power in resistance trained males. Twenty-five resistance trained men were randomly assigned to a deadlift group $(D E ; n=14 ;$ age $=24.3 \pm 4.1 \mathrm{y}$; body mass $=84.8 \pm 14.2 \mathrm{~kg}$; body height $=180.3 \pm 6.8 \mathrm{~cm}$ ) or to a squat group ( $S Q ; n=11$; age $=22.3 \pm 1.6 \mathrm{y}$; body mass $=83.0 \pm 13.6 \mathrm{~kg}$; body height $179.9 \pm 6.1 \mathrm{~cm})$. Both groups trained 3 times per week for 6 weeks. The deadlift and the squat were the only lower body maximal strength exercises performed by $D E$ and $S Q$ groups, respectively, while both training programs included jumps. A significantly $(p=0.017)$ greater increase in deadlift $1 R M$ was observed in the DE compared to the SQ group, while the $S Q$ group obtained a significantly $(p=0.049)$ greater increase in squat $1 R M$. A significant increase in jump performance $(p=0.010)$, without significant interactions between groups $(p=0.552)$, was observed in both groups. Three participants of the DE group developed lower back pain and were excluded from the study. Results indicate that both the squat and the deadlift can result in similar improvement in lower body maximal strength and jump performance and can be successfully included in strength training programs. The incidence of back pain in the DE group may suggest a marked stress of this exercise on the lower back. Proper technique should be used to minimize the risk of injury, especially when the deadlift is performed.
\end{abstract}

Key words: resistance training, jump performance, strength exercises.

\section{Introduction}

The deadlift and the squat are part of the powerlifting competitive program (Chiu, 2007) and are widely included in resistance training to enhance lower body strength and power. The deadlift and the squat involve several muscles of the lower and of the upper body (Bird et al., 2010; Schoenfeld, 2010) and activate both the knee extensors and flexors (Camara et al., 2016). The squat has been shown to enhance power expressed in sprints and jumps and to prevent knee injuries (Ebben et al., 2000; Hartmann et al., 2013). In addition, the squat 1 repetition maximum (1RM) is considered a fundamental assessment of lower body maximal strength (Escamilla, 2001; Escamilla et al., 1998). Recently, the force-power curve has been calculated in the deadlift (Blatnik et al., 2014) and a load of $50 \%$ of the deadlift 1RM has been suggested to be optimal for power development. Both the deadlift and the squat present several similarities when analogue hip and knee joint angles are used. Both exercises extensively activate hip, knee and ankle extensors as well as spine erectors (Hales et al., 2009). The squat and the deadlift, however, also present some dissimilarities. The sticking point, characterized by a reduction in the lifting speed, occurs in correspondence with different hip, knee and ankle positions in the deadlift and the squat (Hales et al., 2009). In addition, different contraction timing of prime movers has been detected by the same authors (Hales et al., 2009). Differences between the two exercises are mainly

1 - Department for Life Quality Studies, University of Bologna, Italy. 
related to the bar position, which markedly influences lifting techniques. The deadlift is characterized by angles between 66 and 149 degrees for the hips, and between 57 and 95 degrees for the knees (Hales et al., 2009). These variables are influenced by individual anthropometric characteristics since the bar height remains constant. On the contrary, the squat can be performed using several different depths. According to Drinkwater et al. (2012), knee angles of 60 and 90 degrees characterize the parallel and the half squat, respectively. The deadlift indeed, is characterized by a higher knee flexion compared to the half squat, but by a lower knee flexion compared to the full squat. Athletes competing in powerlifting events are usually able to lift greater loads in the deadlift compared to the parallel squat. The lower knee flexion in the deadlift compared to the parallel or the full squat, is compensated by relevant loads on the osteoarticular and ligament structures of the trunk (Solomonov et al., 1987).

Previous investigations have reported positive effects of both the deadlift and the squat on vertical jump performance, especially when ballistic contractions were also included in the training program (Mangine et al., 2008).

Despite the popularity of both exercises among strength and power athletes, to the best of our knowledge no experimental studies to date have compared the effects of these two exercises on lower body maximum strength and power.

Thus, the aim of the present investigation was to compare the effects of two strength training programs including either the deadlift or the parallel squat on lower body strength and power in young, resistance trained men. The secondary aim of the present study was to compare the effects of the deadlift and the squat on lower body muscle hypertrophy.

\section{Methods}

\section{Participants}

Twenty-five resistance trained men with more than 3 years of training experience (minimum 3 days/week) participated in the present study. They were randomly assigned to a DE group $(\mathrm{n}=14$; age $=24.3 \pm 4.1 \mathrm{y}$; body mass $=$ $84.8 \pm 14.2 \mathrm{~kg}$; body height $=180.3 \pm 6.8 \mathrm{~cm}$; squat $1 \mathrm{RM}=124.0 \pm 15.6 \mathrm{~kg}$; DE $1 \mathrm{RM}=129.1 \pm 35.2 \mathrm{~kg}$ ) or to a SQ group $(\mathrm{n}=11$; age $=22.3 \pm 1.6 \mathrm{y}$; body mass $=83.0 \pm 13.6 \mathrm{~kg}$; body height $=179.9 \pm 6.1 \mathrm{~cm}$; squat $1 \mathrm{RM}=152.7 \pm 29.7 \mathrm{~kg}$; deadlift $1 \mathrm{RM}=141.4$ $\pm 27.1 \mathrm{~kg}$ ). All participants volunteered to take part in this investigation. Inclusion criteria required participants to be between the ages of 18 and $35 \mathrm{y}$ and have competed in strength and power events in the past 2 yrs prior to the study. Participants were collegiate Track and Field throwers, Division II Rugby players, or Division II American Football players ( $\mathrm{n}=15,5$ and 5, respectively) competing in the Italian championships. Participants were not permitted to use any dietary supplements and did not consume any androgens or other performance enhancing drugs. Screening for performance enhancing drug use and additional supplementation was accomplished via a health questionnaire completed at the recruitment stage. The study was approved by the University's Ethical Committee. Testing procedures were fully explained to each participant before obtaining individual written informed consent.

\section{Design and Procedure}

Participants were randomly assigned to a SQ group including only the squat as a lower body strength exercise or to a DE group including only the deadlift as a lower body resistance exercise. Before and after a 6-week training period, participants were assessed for maximal strength and power, also anthropometric measures were taken. Both training programs consisted of the same upper and lower body exercises, with the exception of the deadlift and the squat exercises. In addition, both training programs were characterized by the same total training volume calculated as the number of repetitions $\mathrm{x}$ sets $\mathrm{x} \%$ of 1RM (Bartolomei et al., 2014).

Testing procedures were fully explained to each participant before obtaining individual written informed consent. Anthropometric evaluations were performed prior to performance assessments. A standardized warm-up (Bartolomei et al., 2018a) consisting of $5 \mathrm{~min}$ jogging, 10 body weight squats, 10 body weight walking lunges, 10 dynamic walking hamstring stretches, 10 dynamic walking quadriceps stretches and 10 body weight push-ups was performed before the evaluations. All the assessments were supervised by qualified investigators. 


\section{Training Protocols}

Both training programs included 3 training sessions per week for 6 consecutive weeks. Both groups performed the same upper body exercises, with participants in the SQ group performing the parallel squat, and participants in the DE group performing the deadlift. The squat was characterized by a position in which the inguinal crease was in projection with the top of the knee (Hartmann et al., 2013; Wretenberg et al., 1996) in the eccentric phase, while the DE program was characterized by full hip extension following the concentric phase. Both the squat and the deadlift exercises were performed using a tempo characterized by a $2 \mathrm{~s}$ eccentric/concentric phase, with no break in the transition phase, and no rest before the next repetition (Wilk et al., 2018). Jumps were included in both training programs following the squat or the deadlift exercises. All exercises performed in both the SQ and DE training programs are reported in Table 1. The number of sets performed in both programs was constant during the training period while training intensity increased throughout the training weeks. Resistance training paradigms of both the SQ and the DE groups are reported in Table 2. All exercises included in both training programs were performed using the same paradigm and the same tempo as the squat and the deadlift exercises. The average training volume in each training session was $9975.3 \pm$ $3221.1 \mathrm{~kg}$ and the total training volume of an average participant during the 6-week program was about $179950 \mathrm{~kg}$.

\section{Anthropometric Measurements}

Participants performed anthropometric assessments before (pre) and after (post) the 6week training period. Body composition was estimated using the three-site skin caliper method (Evans et al., 2005). Fat free mass (FFM) and fat mass (FM) were then calculated. In addition, the thigh cross sectional area (TCSA) was estimated using the following equation (Housh et al., 2014):

TCSA $\left(\mathrm{cm}^{2}\right)=(4.68 x$ thigh circumference in $\mathrm{cm})-$ (2.09 $x$ thigh skinfold in $\mathrm{mm})-80.99$

Thigh circumference and its skinfold were measured halfway between the inguinal fold and the superior margin of the patella. Body mass and height were also determined to the nearest $0.1 \mathrm{~kg}$ and $0.1 \mathrm{~cm}$, using a stadiometer and a scale, respectively (Seca 769, Seca Scale Corp., Munich,
Germany). All measurements were repeated pre and post the training period.

\section{Strength and Power Measurements}

Following a standardized warm-up, participants were asked to perform a counter movement jump (CMJ) test on a contact mat (Globus ergo jump, Globus inc, Treviso, Italia). Each participant performed three attempts with a 3 min rest interval between each jump. Peak power (CMJP) was calculated using the following equation (Aagaard et al., 2001):

\section{Peak Power $=60.7 \mathrm{x}$ jump height $+45.3 \mathrm{x}$ body mass $-\mathbf{2 . 0 5 5}$}

The intraclass coefficient for CMJP was 0.91 (SEM $=106.9 \mathrm{~W}$ ).

\section{Maximal strength assessments}

Maximal strength assessments consisted of an isometric midthigh pull (IMTP) on a power rack that permitted fixation of the bar at a height that corresponded to the participant's midthigh while standing on a force plate (Kisler, Winterthur, Switzerland, $500 \mathrm{~Hz}$ ). Participants were instructed to assume a body position as in the second pull of the clean and jerk. The knee angle, hip angle, and grip width were measured to reproduce the same position for all testing sessions. Participants were secured to the bar using lifting straps and subsequently performed 2 maximal isometric pulls lasting $6 \mathrm{~s}$ with a rest interval of $3 \mathrm{~min}$ between the attempts. Peak force $(\mathrm{PF})$ and the peak rate of force development (pRFD) were calculated using a $50 \mathrm{~ms}$ window, as suggested by Haff et al. (1997). Intraclass coefficients were $0.92(\mathrm{SEM}=100.9 \mathrm{~N})$ and 0.80 $(\mathrm{SEM}=1181.3 \mathrm{~N} / \mathrm{s})$ for PF and pRFD, at the IMTP, respectively. Following maximal isometric strength assessments, 1-RM tests for the squat and the deadlift were performed in randomized order, using the procedure previously described by Hoffman (2014). Briefly, each participant performed two warm-up sets using a resistance of approximately $40-60 \%$ and $60-80 \%$ of his perceived maximum, respectively. For each exercise, 3-4 subsequent trials were performed to determine the 1-RM. A 3-5 min rest interval was provided between each trial. Trials in which the range of motion or exercise technique was not satisfactory were discarded. All strength and power assessments were repeated at the end of the training program. 


\section{Statistical Analysis}

The Shapiro Wilks test was used to test for normal distribution of the data. Data were statistically analysed using a group (SQ $\times$ DE) by time (PRE $x$ POST) repeated measures analysis of variance. Repeated dependent T-tests were used as post hoc analysis when significant interaction between groups were found. The level of significance was set at $p \leq 0.05$. Mean percentage change values ([POST mean - PREmean]/ [PREmean] $x$ 100) were evaluated with $95 \%$ confidence intervals. Relationships between changes in performance and anthropometric measures were calculated using Pearson's correlation coefficients. All data are reported in Tables as mean \pm SD .

\section{Results}

Anthropometric changes between PRE and POST for both the SQ and the DE group are presented in Table 3. No significant interactions between the two groups were noted for FM $(\mathrm{F}=$ 0.232; $\left.p=0.573 ; \eta^{2}=0.012\right)$, FFM $(\mathrm{F}=0.513 ; p=$ $\left.0.482 ; \eta^{2}=0.025\right)$ and for TCSA $(F=0.225 ; p=0.742$; $\left.\eta^{2}=0.006\right)$. A significant main effect of time was detected for FFM $\left(\mathrm{F}=11.14 ; p=0.003 ; \eta^{2}=0.358\right)$. No significant main effects of time were detected for FM ( $\left.F=0.252 ; p=0.621 ; \eta^{2}=0.012\right)$ and for TCSA $\left(\mathrm{F}=0.052 ; p=0.821 ; \eta^{2}=0.003\right)$.

Results for strength and power are reported in Table 4. Significant interactions between groups were detected for squat $1 \mathrm{RM}(\mathrm{F}=$ 4.421; $\left.p=0.049 ; \eta^{2}=0.241\right)$ and deadlift $1 \mathrm{RM}(\mathrm{F}=$ $\left.6.843 ; p=0.017 ; \eta^{2}=0.255\right)$. The SQ group improved 1RM squat performance by an average of $15.2 \%$, while the DE group improved by an average of $5.7 \%$. On the contrary, improvements by a mean of $17.7 \%$ were detected in the deadlift $1 \mathrm{RM}$ in the DE group, while it equaled $6.7 \%$ in the
SQ group. No interactions between groups were detected for PF and pRFD expressed at the IMTP $\left(\mathrm{F}=0.349 ; p=0.563 ; \eta^{2}=0.021\right.$ and $\mathrm{F}=0.042 ; p=$ $0.839 ; \eta^{2}=0.003$, for PF and pRFD, respectively). No significant interactions between groups were found for CMJP ( $\left.\mathrm{F}=0.367 ; p=0.552 ; \eta^{2}=0.019\right)$. Significant main effects of time were detected for the squat $1 \mathrm{RM}\left(\mathrm{F}=27.030 ; p<0.01 ; \eta^{2}=0.575\right)$; deadlift 1RM ( $\left.\mathrm{F}=28.910 ; p<0.01 ; \eta^{2}=0.591\right)$ and CMJP $\left(F=8.146 ; p=0.010 ; \eta^{2}=0.300\right)$. No significant main effects were detected for PF and pRFD expressed at the IMTP $(\mathrm{F}=0.514 ; p=0.484$; $\eta^{2}=0.031$ and $F=0.557 ; p=0.446 ; \eta^{2}=0.034$, respectively). Increases in the CMJP were significantly correlated with improvements in FFM $(\mathrm{r}=0.90 ; p<0.001)$; in the SQ 1RM $(\mathrm{r}=0.87 ; p$ $<0.001)$ and in the deadlift 1RM ( $\mathrm{r}=0.71 ; p=$ 0.001). Three participants in the DE group developed lower back pain and withdrew from the study within the first four weeks of training.

\section{Discussion}

Results of the present study indicate that different strength training programs in which either the deadlift or the parallel squat was the only lower-body resistance exercise elicited similar increases in lower body power $(+4.9 \%$ and $+5.5 \%$, for the DE and the SQ group, respectively). Despite significant improvements in maximal strength and power following both training programs, no significant increases in TCSA were detected. The duration of the training program may not be sufficient to obtain significant increases in muscle hypertrophy even if lower limbs were trained 3 times per week. Curiously, a general increase in FFM was detected in both groups.

\section{Table 1}

Exercises for both $D E$ and $S Q$ groups.

\begin{tabular}{ccc}
\hline Monday & Thursday & Friday \\
\hline Squat or Deadlift & Squat or Deadlift & Squat or Deadlift \\
CMJ & CMJ & CMJ \\
Bent Over Row & Bench Press & Incline Bench Press \\
Lat Machine & Military Press & Standing Upright Row \\
Standing Biceps Curl & Lateral Raises & French Press \\
Leg Curl & \\
\hline \multicolumn{2}{c}{ NOTE: CMJ = countermovement jump. }
\end{tabular}


Table 2

Training programs for DE and SQ groups.

\begin{tabular}{cccccc}
\hline week & Reps & Series & \% 1RM & Jump & Rest \\
\hline $\mathbf{1}$ & 10 & 5 & 70 & $4 \times 8$ & $1^{\prime}$ \\
\hline $\mathbf{2}$ & 8 & 5 & 75 & $4 \times 8$ & $1^{\prime} 20^{\prime \prime}$ \\
\hline $\mathbf{3}$ & 6 & 5 & 80 & $4 \times 6$ & $1^{\prime} 45^{\prime \prime}$ \\
\hline $\mathbf{4}$ & Pyr. $5-4-3-4-5$ & 5 & $80-85-90-85-80$ & $4 \times 6$ & $22^{\prime} 15$ \\
\hline $\mathbf{5}$ & 5 & 5 & 85 & $4 \times 5$ & $2^{\prime} 15$ \\
\hline $\mathbf{6}$ & 4 & 4 & 90 & $4 \times 4$ & $2^{\prime} 30^{\prime \prime}$ \\
\hline
\end{tabular}

NOTE: Pyr = pyramid method.

Table 3

Anthropometric characteristics of DE and SQ groups in PRE and POST assessments.

\begin{tabular}{cccc}
\hline & & $\begin{array}{c}\text { SQ group } \\
(\text { mean } \pm \text { DS })\end{array}$ & $\begin{array}{c}\text { DE group } \\
(\text { mean } \pm \text { DS })\end{array}$ \\
\hline \multirow{2}{*}{ FFM $(\mathbf{k g})$} & PRE & $72.5 \pm 7.9$ & $74.9 \pm 7.7$ \\
\cline { 2 - 4 } & POST\# & $73.1 \pm 8.1$ & $75.9 \pm 8.1$ \\
\hline \multirow{2}{*}{ FM (kg) } & PRE & $10.5 \pm 7.0$ & $10.2 \pm 6.5$ \\
& POST & $10.5 \pm 6.8$ & $10.0 \pm 8.3$ \\
\hline TCSA (cm $\left.{ }^{2}\right)$ & PRE & $163.5 \pm 13.2$ & $168.1 \pm 13.8$ \\
\cline { 2 - 4 } & POST & $163.3 \pm 14.3$ & $169.0 \pm 14.4$ \\
\hline
\end{tabular}

NOTE: FFM = Fat Free Mass; FM =Fat Mass; TCSA = Thigh Cross Sectional Area; \# indicates a significant time effect $(p<0.05)$.

\section{Table 4}

Strength and power assessment of $S Q$ and DE groups.

\begin{tabular}{|c|c|c|c|}
\hline & & $\begin{array}{l}\text { SQ group } \\
(m e a n \pm D S)\end{array}$ & $\begin{array}{c}\text { DE group } \\
(\text { mean } \pm D S)\end{array}$ \\
\hline \multirow[t]{2}{*}{ Squat 1RM (kg) } & PRE & $124.0 \pm 15.6$ & $152.7 \pm 29.7$ \\
\hline & POST \# & $140.5 \pm 18.8^{*}$ & $160.0 \pm 34.6$ \\
\hline \multirow[t]{2}{*}{ Deadlift 1RM (kg) } & PRE & $129.1 \pm 35.2$ & $141.4 \pm 27.1$ \\
\hline & POST\# & $137.7 \pm 32.2$ & $166.4 \pm 29.7^{*}$ \\
\hline \multirow[t]{2}{*}{ PF at IMTP (N) } & PRE & $1781.1 \pm 448.6$ & $1831.7 \pm 333.0$ \\
\hline & POST & $1785.5 \pm 378.6$ & $1876.9 \pm 256.4$ \\
\hline \multirow{2}{*}{$\begin{array}{c}\text { pRFD at IMTP } \\
\left(\mathrm{N} / \mathrm{s}^{-1}\right)\end{array}$} & PRE & $8733.5 \pm 2743.1$ & $10724.2 \pm 711.7$ \\
\hline & POST & $8954.0 \pm 2200.3$ & $11112.5 \pm 2326.2$ \\
\hline \multirow[t]{2}{*}{ CMJP (W) } & PRE & $4291.3 \pm 569.1$ & $4406.2 \pm 512.7$ \\
\hline & POST\# & $4407.5 \pm 562.3$ & $4487.7 \pm 587.7$ \\
\hline
\end{tabular}

NOTE: PF = peak force; IMTP = Isometric Mid-Thigh Pull;

$C M J P=$ countermovement jump power; $p R F D=$ peak rate of force development; * indicates a significant interaction between the groups $(p<0.05)$; \# indicates a significant time effect $(p<0.05)$. 
The lack of significant changes in TCSA indicate that the increase in muscle mass mainly involved the upper body. Improvements in maximum strength and power without significant increases in lower body muscle mass, suggest that nervous adaptations may have occurred.

Despite that both training programs led to a significant improvement in jump performance, no significant interactions between groups were noted for the CMJP. Jump performance was significantly correlated with isometric maximum strength measured at the mid-thigh pull, while it was not significantly correlated with other variables of explosive strength such as the pRFD. Poor correlations between the inclination of the force-time curve measured in the deadlift or in the squat exercise and performance in the $\mathrm{CMJ}$ have been previously reported by several authors (Adams et al., 1992; Nuzzo et al., 2008; Weeks et al., 2011; Wisloff et al., 2004; Zemkova 2019). As suggested by Hoffman (2014), the execution of the mid-thigh pull may be affected by the participants explosive intent. In particular, the pRFD may be negatively influenced by a poor technique or by a submaximal activation at the beginning of the pull (Bartolomei et al., 2018b).

The correlations found in this study between the 1RM deadlift and 1RM squat $(r=$ $0.60)$ are comparable to those reported by other authors (Ebben et al., 2008). Furthermore, in the present investigation, a significant correlation in jump power was detected in both groups. This confirms the relationship between maximum strength and power suggested by several authors (Chiu, 2007; Haff et al., 1997). In addition, the present study confirms that improvements in maximal strength of lower body can lead to greater lower body power performances, especially when jumping exercises are included in the training routine (Chadler and Stone, 1991; Harris et al., 2000).

Three participants in the DE group reported back pain within the first four weeks of training and withdrew from the study. A greater stress on the lumbar spine may be induced by the deadlift exercise compared to the squat. The biomechanics of the deadlift may result in a more accentuated anterior tilt of the trunk than the squat exercise with a consequent functional overload of the back extensors (Rippetoe et al., 2007). The typical condition of exhaustion reached in the last repetitions of each set may have affected technique as previously reported by Trafimow et al. (1993). Technique should be prioritized especially when the deadlift is performed using heavy loads (Flis-Masłowska et al., 2014). On the contrary, no participants in the SQ group reported back pain.

In conclusion, the present study suggests that both the deadlift and the squat can be successfully included in training programs to obtain significant gains in both lower body maximal strength and power when associated with plyometric exercises. Increases in maximal strength and FFM are related to the improvements in lower limb power. Athletes and coaches, however, should be aware that lower back pain may occur using heavy loads in the deadlift. To avoid injuries, a correct technique should be maintained even when repetitions to exhaustion are performed.

Limitations of the present investigation include a short training period duration, and the lack of morphological assessment of upper and lower body muscles (e.g. ultrasound), pre and post both training programs.

\section{References}

Aagaard P, Andersen JL, Dyhre-Poulsen P, Leffers AM, Wagner A, Magnusson SP, Simonsen EB. A mechanism for increased contractile strength of human pennate muscle in response to strength training: changes in muscle architecture. J Physiol, 2001; 534(2): 613-623

Adams K, O'Shea JP, O'Shea KL, Climstein M. The effect of six weeks of squat, plyometric and squatplyometric training on power production. J Strength Cond Res, 1992; 6(1): 36-41

Bartolomei S, Hoffman JR, Merni F, Stout JR. A comparison of traditional and block periodized strength training programs in trained athletes. J Strength Cond Res, 2014; 28(4): 990-7

Bartolomei S, Hoffman JR, Stout JR, Merni F. Effect of Lower-Body Resistance Training on Upper-Body Strength Adaptation in Trained Men. J Strength Cond Res, 2018a; 32(1): 13-18 
Bartolomei S, Nigro F, Gubellini L, Semprini G, Ciacci S, Hoffman JR, Merni F. Acute Effects of Ammonia Inhalants on Strength and Power Performance in Trained Men. J Strength Cond Res, 2018b; 32(1): 244247

Bird S, Barrington-Higgs B. Exploring the deadlift. Strength Cond J, 2010; 32(2): 46-51

Blatnik JA, Goodman CL, Capps CR, Awelewa OO, Triplett TN, Erickson TM, McBride JM. Effect of load on peak power of the bar, body and system during the deadlift. J Sports Sci Med, 2014; 13(3): 511

Camara KD, Coburn JW, Dunnick DD, Brown LE, Galpin AJ, Costa PB. An examination of muscle activation and power characteristics while performing the deadlift exercise with straight and hexagonal barbells. J Strength Cond Res, 2016; 30(5): 1183-1188

Chandler TJ, Stone MH. A Position Statement and Literature Review of the Squat Exercise in Athletic Conditioning. Colorado Springs, CO. National Strength and Conditioning Association; 1992

Chiu LZF. Powerlifting versus weightlifting for athletic performance. Strength Cond J, 2007; 29(5): 55-57

Ebben WP, Feldmann CR, Dayne A, Mitsche D, Chmielewsky M, Alexander P, Knetgzer KJ. Using squat testing to predict training loads for the deadlift, lunge, step-up and leg extension exercises. J Strength Cond Res, 2008; 22(6): 1947-1949

Ebben WP, Randall LJ. The role of back squat as an harmstring training stimulus. Strength Cond J, 2000; 22(5): 15-17

Escamilla RF, Fleising GS, Zheng N, Barrentine SW, Wilk KC, Andrew JR. Biomechanics of the knee during closed kinetic chain and open kinetic chain exercises. Med Sci Sports Exerc, 1998; 30: 556-569

Escamilla RF. Knee biomechanics of the dynamic squat exercise. Med Sci Sports Exerc, 2001; 33: 127-141

Evans EM, Rowe DA, Misic MM, Prior BM, Arngrímsson SA. Skinfold prediction equation for athletes developed using a four-component model. Med Sci Sports Exerc, 2005; 37(11): 2006-2011

Flis-Masłowska M, Trzaska T, Wiernicka M, Lewandowski J. Structural lesions in the lumbosacral spine in field hockey players. Sports Med, 2014; 67: 473-484

Haff, GG, Stone M, O'bryant HS, Harman E, Dinan C, Johnson R, Han KH. Force-time dependent characteristics of dynamic and isometric muscle actions. J Strength Cond Res, 1997; 11(4): 269-272

Hales ME, Johnson BF, Johnson JT. Kinematic Analysis of the Powerlifting Style Squat and the Conventional Deadlift During Competition: Is There a Cross-Over Effect Between Lifts? J Strength Cond Res, 2009; 23(9): 2574-2580

Harris G, Stone M, O'Bryant H, Proulx C, Johnson R. Short-Term Performance Effects of High Power, High Force, or Combined Weight-Training Methods. J Strength Cond Res, 2000; 14(1)

Hartmann H, Wirth K, Klusemann M. Analysis of the load on the knee joint and vertebral column with changes in squatting depth and weight load. Sports Med, 2013; 43(10): 993-1008

Hoffman JR. Physiological aspects of sport training and performance. Champaign, IL. Human Kinetics; 2014

Housh DJ, Housh TJ, Weir JP, Weir LL, Johnson GO, Stout JR. Anthropometric estimation of thigh crosssectional area. Med Sci Sports Exerc, 2014; 27(5): 789-791

Mangine GT, Ratamess NA, Hoffman JR, Faigenbaum AD, Kang J, Chilakos A. The effect of combined ballistic and heavy resistance training on maximal lower- and upper-body strength in recreationally trained man. J Strength Cond Res, 2008; 22(1): 132-139

Nuzzo JL, McBride JM, Cormie P, McCaulley GO. Relationship between countermovement jump performance and multijoint isometric and dynamic tests of strength. J Strength Cond Res, 2008; 22(3): 699-707

Rippetoe M, Kilgore L. Starting strength: Basic barbell training. Wichita Falls, Texas, USA: Aasgaard Company; 2007

Schoenfeld BJ. Squatting kinematics and kinetics and their application to exercise performance. J Strength Cond Res, 2010; 24(12): 3497-3506

Solomonov M, Baratta R, Zhou BH, Shoji H, Bose W, Beck C. The synergistic action of the anterior cruciate ligament and thigh muscles in maintaining joint ability. Am J Sports Med, 1987; 15: 207-213

Trafimow J, Schipplein OD, Novak GJ, Andersson GBJ. The effects of quadriceps fatigue on the technique of lifting. Spine, 1993; 18: 364-367 
Weeks C, Trevino J, Blanchard G, Kimpel S. Effect of squat depth training on vertical jump performance. J Strength Cond Res, 2011; 25(1): 32-33

Wilk M, Golas A, Stastny P, Nawrocka M, Krysztofik AZ. Does tempo of resistance exercise impact training volume? J Hum Kinet, 2018; 62, 241-250

Wisloff U, Castagna C, Helgerud J, Hoff J. Strong correlation of maximal squat strength with sprint performance and vertical jump height in elite soccer players. British J Sports Med, 2004; 38(10): 285-288

Wretenberg PER, Feng YI, Arborelius UP. High-and low-bar squatting techniques during weight-training. Med Sci Sports Exerc, 1996; 28(2): 218-224

Zemkova E. Reliability of a novel method assessing muscle power and velocity during seated trunk rotations. Phys Activ Rev, 2019, 7: 1-8

\section{Corresponding author:}

\section{Sandro Bartolomei}

University of Bologna

Department for Life Quality Studies

Via del Pilastro 8

40100 Bologna, Italy

051-2088777

E-mail: sandro.bartolomei@unibo.it 